\title{
Repeated iodine-125 seed implantations combined with external beam radiotherapy for the treatment of locally recurrent or metastatic stage III/IV non-small cell lung cancer: a retrospective study
}

\author{
Wei Li, Gang Dan, Jianqing Jiang, Yifeng Zheng, Xiushan Zheng and Dan Deng*
}

\begin{abstract}
Background: Recurrent or metastatic lung cancer is difficult to manage. This retrospective study aimed to assess the efficacy of repeated iodine-125 seed implantations combined with external beam radiotherapy (EBRT) for locally recurrent or metastatic stage-III/IV non-small cell lung cancer (NSCLC).

Methods: Eighteen previously treated stage-III/IV NSCLC patients with local or metastatic recurrences underwent 1to-3 iodine-125 implantations. Six of these patients received palliative EBRT and six patients received combined chemotherapy using gemcitabine and cisplatin. Near-term treatment efficacy was evaluated 3 months after seed implantation by comparing changes in tumor size on computed tomography images; the evaluated outcomes were complete response, partial response, stable disease, and local tumor control rate. Long-term efficacy was assessed based on 1- and 2-year survival rates.
\end{abstract}

Results: Patients were followed up for 6 to 50 months. The overall (i.e., complete + partial) response rate was 87 . $4 \%$. The local control rates after the first, second, and third years were 94.1, 58.8 and $41.2 \%$, respectively.

Conclusions: The results of this study demonstrated that repeated implantation of radioactive particles combined with EBRT is a safe treatment that effectively controlled local recurrence and metastasis of stage III/IV NSCLC.

Keywords: Non-small cell lung cancer, $1^{125}$ seed implantation, External beam radiotherapy, Metastasis, Recurrence, Brachytherapy

\section{Introduction}

Lung cancer is the leading cause of cancer death in both men and women worldwide, and its prevalence is increasing [1]. Conventional treatment for lung cancer includes palliative radiotherapy and chemotherapy, which has shown limited potential in increasing long-term survival rates [2]. Using external beam radiotherapy (EBRT) alone, it is difficult to deliver a sufficient radiation dose to patients with

\footnotetext{
* Correspondence: 348418684@qq.com

Department of Thoracic Surgery, PLA Chengdu Military General Hospital, Chengdu 610083, People's Republic of China
}

large or advanced tumors, in order to avoid damage to adjacent normal tissues [3]. Repeated EBRT has limited efficacy for these patients and is associated with increased side effects because of the low tolerance of the surrounding normal tissue [4]. Furthermore, because a large proportion of lung cancers are diagnosed at an advanced stage, the main challenges to treatment are recurrence and metastasis, which is difficult to manage. Extensive resection of a locally recurrent lung cancer is associated with high recurrence risk and significant mortality. Mediastinal metastasis and metastasis in the brain, bone, or distant

(c) 2016 The Author(s). Open Access This article is distributed under the terms of the Creative Commons Attribution 4.0 International License (http://creativecommons.org/licenses/by/4.0/), which permits unrestricted use, distribution, and reproduction in any medium, provided you give appropriate credit to the original author(s) and the source, provide a link to the Creative Commons license, and indicate if changes were made. The Creative Commons Public Domain Dedication waiver (http://creativecommons.org/publicdomain/zero/1.0/) applies to the data made available in this article, unless otherwise stated. 
organs are common in patients with advanced lung cancer [5]. Controlling the size of these metastatic tumors may help to improve long-term survival rate.

Radioactive seed implantation, which is a form of brachytherapy, can be performed repeatedly. It has been successfully applied for treating inoperable solitary lung cancers, while avoiding excessive radiation exposure to surrounding tissues [6-8]. Clinically, brachytherapy using iodine-125 seed implantation is capable of delivering a sufficient dose of radiation to the tumor mass. However, in many recurrent and metastatic lung cancers, important organs, large vessels, or bone structures often block the pathway to the lesions, making these tumors inaccessible to needle puncture, and leading to an unsatisfactory distribution of the implanted iodine- 125 seeds $[9,10]$. In addition, brachytherapy would become very risky when conducting implantation in metastatic lymph nodes located near the mediastinal macrovascular area.

In this retrospective study, we investigated the efficacy of combining iodine-125 seed implantations with EBRT for salvage treatment of advanced lung cancers. Eighteen recurrent advanced non-small cell lung cancer (NSCLC) patients who previously underwent iodine-125 seed implantations received computed tomography (CT)-guided iodine-125 seed reimplantations in combination with EBRT.

\section{Materials and methods}

\section{Patients}

The Institutional Review Board and Ethics Committee of General Hospital of Chengdu Military Region of the PLA (\#08-00125) approved this study. All patients provided written informed consent.

Between October 2006 and September 2014, 18 patients with recurrent or metastatic stage III/IV NSCLC (Table 1) underwent CT-guided iodine-125 seed reimplantations, with or without EBRT, at the General Hospital of Chengdu Military Region of PLA. All of the 18 patients enrolled in this study had a history of iodine- 125 seed implantations and met the following criteria: NSCLC was confirmed by histopathological examination; a CT scan indicated a solid mass or nodule in the lung area, or nearby the mediastinum; local recurrence or restricted metastasis at $<3$ regions; the Karnofsky Performance Status score was $\geq 60$.

\section{Treatment}

Among the 18 patients evaluated (Tables 1 and 2), two patients with vertebral metastasis (Patients 8 and 17) and great pain and one patient with metastasis in the lymph nodes of the mediastinum (Patient 13) received palliative EBRT at 20-30 Gy. In addition, three patients with mediastinum metastasis (Patients 5, 10, and 18) received palliative EBRT at the lymph nodes of the mediastinum. Two patients with brain

Table 1 Clinical features of the present 18-patient cohort

\begin{tabular}{|c|c|c|c|c|c|c|c|c|}
\hline No. & Age, y & Gender & TNM & $\mathrm{KPS}^{\mathrm{a}}$ & Tumors & Size, $\mathrm{cm}^{3}$ & Seeds ${ }^{b}$; activity, mCi & MPD, Gy \\
\hline 1 & 72 & M & T3N1M0 & 70 & $L$, lung SCC & $6 \times 5 \times 5$ & $72 / 59 / 43 ; 0.7-0.8$ & 130 \\
\hline 2 & 57 & $\mathrm{~F}$ & T3N1M0 & 80 & R, lower lung adenocarcinoma & $6 \times 6 \times 5$ & $76 / 45 / 52 ; 0.8$ & 140 \\
\hline 3 & 59 & M & T3N1M1 & 70 & $L$, lower lung adenocarcinoma & $6 \times 5 \times 4$ & $77 / 20 ; 0.7$ & 140 \\
\hline 4 & 59 & M & T3N2M0 & 70 & $L$, lung SCC & $5 \times 5 \times 4$ & $66 / 62 ; 0.8$ & 150 \\
\hline 5 & 73 & M & $\mathrm{T} 2 \mathrm{~N} 2 \mathrm{MO}$ & 80 & R, lower lung adenocarcinoma with mediastinal LNM & $6 \times 7 \times 6$ & $63 / 24 ; 0.8$ & 140 \\
\hline 6 & 70 & M & T3N1M1 & 70 & $\mathrm{~L}$, lung SCC with brain metastases & $5 \times 5 \times 4$ & $45 ; 0.7$ & 130 \\
\hline 7 & 54 & M & T3N1M0 & 60 & L, lung poorly differentiated SCC & $8 \times 7 \times 5$ & $100 / 40 ; 0.6$ & 150 \\
\hline 8 & 81 & M & $\mathrm{T} 3 \mathrm{~N} 1 \mathrm{MO}$ & 70 & R, lower lung adenocarcinoma with vertebral metastases & $6 \times 5 \times 5$ & $65 / 45 ; 0.8$ & 140 \\
\hline 9 & 66 & $\mathrm{~F}$ & T4N1M0 & 80 & R, lower lung SCC & $6 \times 5 \times 4$ & $70 / 62 ; 0.7$ & 130 \\
\hline 10 & 67 & M & T2N1M1 & 70 & R, upper lung SCC with LNM & $4 \times 4 \times 3$ & $48 / 20 / 14 ; 0.8$ & 130 \\
\hline 11 & 73 & M & $\mathrm{T} 2 \mathrm{~N} 1 \mathrm{MO}$ & 60 & $L$, lung SCC in situ recurrence after 1 y & $5 \times 5 \times 4$ & $62 / 40 ; 0.8$ & 140 \\
\hline 12 & 78 & $\mathrm{~F}$ & T2NOMO & 70 & R, upper lung squamous & $3 \times 1 \times 2$ & 23/40; 0.7 & 140 \\
\hline 13 & 65 & $F$ & $\mathrm{~T} 4 \mathrm{~N} 2 \mathrm{MO}$ & 70 & SCC with mediastinal lymph node metastases & $7 \times 6 \times 6$ & $89 ; 0.7$ & 130 \\
\hline 14 & 75 & M & T3N1M0 & 60 & Adenocarcinoma & $5 \times 6 \times 5$ & 19/40; 0.7 & 130 \\
\hline 15 & 66 & M & $\mathrm{T} 3 \mathrm{~N} 2 \mathrm{M} 1$ & 80 & R, upper lung adenocarcinoma with vertebral metastases & $7 \times 5 \times 4$ & $78 ; 0.6$ & 160 \\
\hline 16 & 60 & $\mathrm{~F}$ & T2N1M1 & 70 & R, upper lung adenocarcinoma with adrenal metastasis & $6 \times 5 \times 5$ & $70 ; 0.8$ & 140 \\
\hline 17 & 57 & M & $\mathrm{T} 4 \mathrm{~N} 1 \mathrm{M} 1$ & 75 & $L$, lung SCC associated with vertebral metastases & $5 \times 5 \times 3$ & $41 / 36 ; 0.8$ & 130 \\
\hline 18 & 64 & M & T3N2M0 & 80 & R, lung moderately differentiated SCC with mediastinal LNM & $4 \times 5 \times 5$ & $52 ; 0.8$ & 120 \\
\hline
\end{tabular}

$L$ left, $L R$ local recurrence, $L N M$ lymph node metastasis, MPD matched peripheral dose, $R$ right, SCC squamous cell carcinoma, $T N M$ tumor, node, metastasis stage

${ }^{a}$ KPS score; ${ }^{b}$ number of iodine-125 seeds per implantation 
Table 2 Recurrence and survival of the present 18-patient cohort

\begin{tabular}{|c|c|c|c|c|c|}
\hline No. & Age, y & Gender & Recurrence/metastasis after first seed implant, further treatment & TTR mo & Survival, mo \\
\hline 1 & 72 & M & LR; 3 implants & $11 / 12$ & 43 \\
\hline 2 & 57 & $\mathrm{~F}$ & LR; 3 implants & $10 / 13$ & 31 \\
\hline 3 & 59 & M & Brain metastases; whole brain irradiation +2 implants & 24 & Living \\
\hline 4 & 59 & M & Adrenal metastasis (R); 2 implants & 13 & Living \\
\hline 5 & 73 & M & LR; 2 implants, yet failed due to hemoptysis & 15 & 15 \\
\hline 6 & 70 & M & - & 12 & Living \\
\hline 7 & 54 & M & LR; 2 implants + mediastinal external irradiation & 11 & 13 \\
\hline 8 & 81 & M & - & 14 & 24 \\
\hline 9 & 66 & $\mathrm{~F}$ & LR; 2 implants & 17 & Living \\
\hline 10 & 67 & M & Neck metastasis; 3 implants & $11 / 13$ & 17 \\
\hline 11 & 73 & M & LR; 2 implants & 14 & Living \\
\hline 12 & 78 & $\mathrm{~F}$ & L; lung metastases at 6 years after first treatment; 2 implants & 78 & Living \\
\hline 13 & 65 & $\mathrm{~F}$ & - & 0 & Living \\
\hline 14 & 75 & M & Neck metastasis; 2 implants & 13 & 16 \\
\hline 15 & 66 & M & - & 16 & 21 \\
\hline 16 & 60 & $\mathrm{~F}$ & Neck metastasis; 1 implant + adrenal EBRT & 0 & 11 \\
\hline 17 & 57 & M & - & 0 & 12 \\
\hline 18 & 64 & M & - & 0 & Living \\
\hline
\end{tabular}

$L R$ local recurrence, $T T R$ time to recurrence

metastasis (Patients 3 and 6) underwent gamma knife radiosurgery.

Seven patients (Patients 2, 5, 7, 11, 14, 16, and 18) received combined chemotherapy using gemcitabine and cisplatin. The regimen had a 21-day schedule during which gemcitabine $\left(1000 \mathrm{mg} / \mathrm{m}^{2}\right)$ was administered on the first and eighth days, and cisplatin $\left(20 \mathrm{mg} / \mathrm{m}^{2}\right)$ on the first, second, and third days. The chemotherapy schedule was repeated for 4 to 6 cycles, if tolerated.

All patients received iodine-125 implantations. Oneto- 2 weeks before iodine- 125 seed implantation, routine blood examination, bleeding time, and coagulation tests were performed to exclude contraindications for needle puncture.

Routine enhanced-CT scans were also performed for assessing tumor volume. Briefly, gross tumor volume was outlined and used as the planning target volume. The minimum matched peripheral dose was set at 110140 Gy. All the implantations were performed in a standard CT room under local anesthesia, and guided by CT using a Fudan TPS 2.00 brachytherapy planning system [11] (Table 1). Iodine-125 seeds with a nominal activity of $0.5-0.7$ millicurie $(\mathrm{mCi})$ per seed and a diameter $<1 \mathrm{~mm}$ were implanted using a turntable implantation gun with 18-G implantation needles (XinKe Pharmaceutical, Shanghai, China), while avoiding puncturing of the nearby vessels and other organs. Patients were kept in the radiation oncology/interventional ward for 1-to-2 days after implantation.
The distribution of the radioactive seeds was evaluated immediately after implantation by $\mathrm{CT}$ scans. Reimplantation was conducted for sites showing an uneven distribution of seeds. The minimum peripheral dose was 110-140 Gy (mean, 120 Gy).

\section{Follow-up and efficacy evaluation}

Each patient underwent a follow-up examination at 1 and 3 months after the seed implantation, and then every 3 months for up to 60 months. Physical examinations, blood tests, and thoracic CT scans were performed. Patients' pain score, the rate of radiation pneumonia, time to recurrence, survival and local control rates, and median survival times were recorded. Survival and locoregional metastasis rates were calculated using the Kaplan-Meier method. For calculation of the survival rate, deaths from any cause were scored as events. Local control was defined as lack of tumor progression in areas adjacent to or at the site of iodine-125 seed implantation and adjacent regions.

To evaluate near-term efficacy after brachytherapy, all patients underwent CT scans 3 months after seed implantation. The total volume of each tumor was normalized to that before implantation. Complete response was defined as the complete disappearance of a lesion for $>4$ weeks. A partial response was considered when the size of the lesion decreased by $>50 \%$, and then remained unchanged for 4 weeks. Stable disease was defined when the size of the tumor decreased by $<50 \%$ or increased 


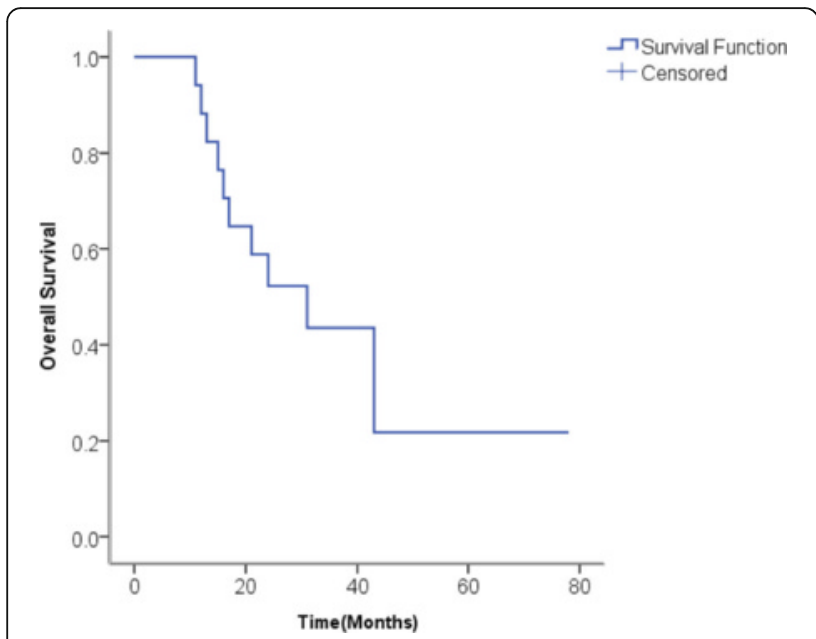

Fig. 1 Kaplan-Meier curve of 18 patients with recurrent or metastatic stage III/IV non-small cell lung cancer

by $<25 \%$. Response rate was defined as the sum of the complete response and partial response. Local tumor control was defined as the absence of tumor progression detected by CT (i.e., stable disease + partial response + complete response). Long-term efficacy was assessed according to 1-year and 2-year survival rates.

\section{Results}

Efficacy

Eighteen patients with recurrent and metastatic advanced NSCLC received a total of 35 implantations of iodine- 125 seeds (Table 1). Based on the imaging results obtained at approximately 2 months after the first implantation, nearterm complete response was achieved in seven cases, partial response in another six cases, and stable disease in five cases. The overall response rate was $72.22 \%$ (i.e., 13/18).

During follow-up after the first round of iodine-125 seed implantation, 12 of the 18 patients experienced tumor recurrence and underwent a second round of iodine-125 seed implantations (Table 2). Four of these 13 patients (Nos. 1, 2, 3, and 10) experienced tumor recurrence again, and underwent a third round of seed implantations. Metastatic tumors were found in the adrenal glands of patient No. 16, and in the vertebra of patient Nos. 8 and 10.

For patient Nos. $6,8,13,15,17$ and 18 , the residual tissues within each tumor mass showed no enhancement on the CT or positron emission tomography (PET)-CT images obtained 3 months after the first round of iodine-125 seed implantation (Table 2). In eight patients, PET-CT scans detected reduced metabolic activity in the lesions or disappearance of the lesions and residual tissues within the tumor mass.
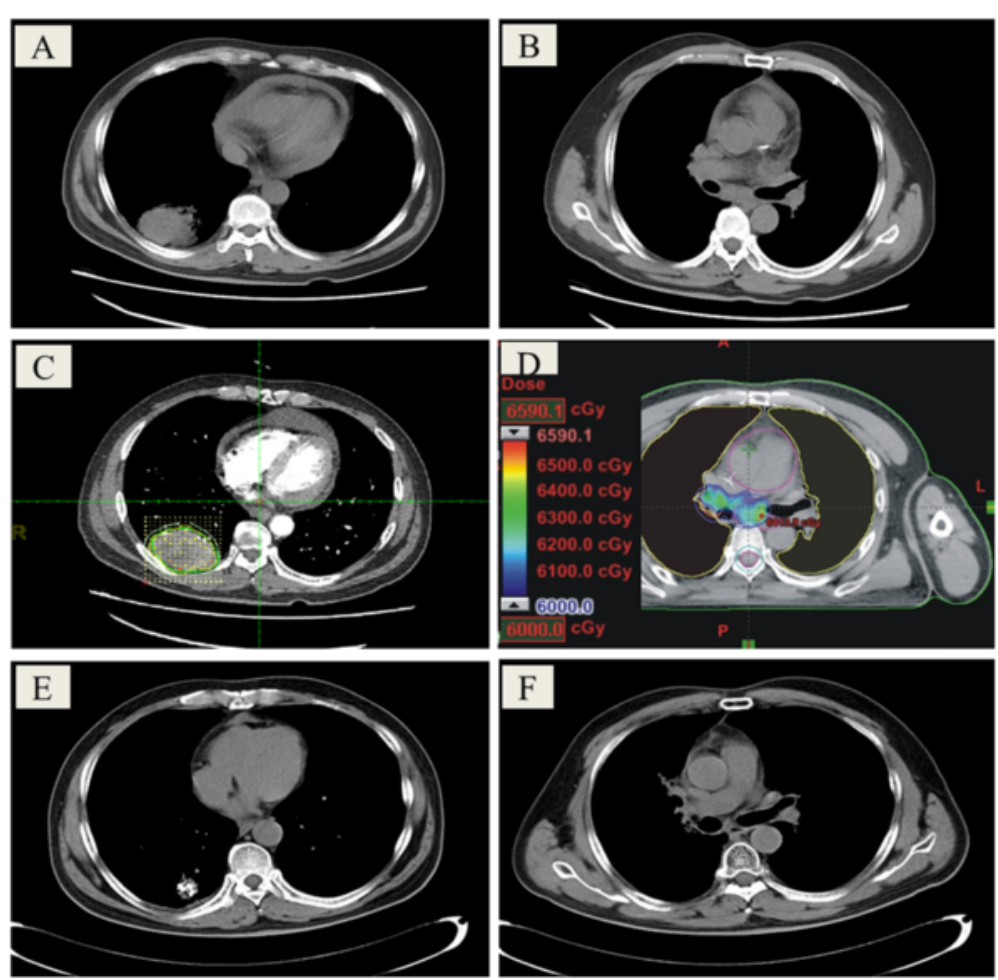

Fig. 2 CT images of moderately differentiated squamous cell carcinoma $\left(4 \times 5 \times 5 \mathrm{~cm}^{3}\right)$ in the lower right lung (a) with metastasis in the lymph nodes of the mediastinum (b) in a 64-year old man (No. 18). The patient received iodine-125 seed implantation for lung tumor (c) and external beam radiotherapy for lymph node metastasis $(\mathbf{d})$. Complete disappearance of the lung tumor $(\mathbf{e})$ and lymph node metastasis (f) was observed 1 month after treatment 
As calculated from the Kaplan-Meier Curve (Fig. 1), the median survival time for this 18-patient cohort was 31 months, and the progression-free survival time was 6-8 months. The overall 1-year and 2-year survival rates were 62.5 and $32.7 \%$, respectively.

Ten patients reported cancer-related pain before treatment. After treatment, complete relief was achieved in all patients with mild pain $(n=4)$, and in two of four patients with moderate pain. Partial relief was achieved in the other two of the four patients with moderate pain, and in one of two patients with severe pain. One patient with severe pain showed no relief. The three patients with partial or no pain relief received steroid-based painkillers.

Two patients (Nos. 7 and 14) experienced rapid tumor recurrence and metastasis in multiple sites after the second implantation, and died of multiple organ failure thereafter.

\section{Adverse reactions and complications}

Complications include pneumothorax and hemoptysis, both are common to puncture. One patient had severe hemoptysis.

The most common complications reported were pain, pneumothorax, and hemoptysis, which are common to puncture. One patient developed grade 2 or higher radiation pneumonitis after the first round of iodine-125 seed implantation. Three patients experienced pneumonitis after the second round of iodine-125 seed implantation. One patient (No. 5) with squamous cell lung carcinoma in the lower right lung experienced tumor recurrence 18 months after the first round of seed implantation. During the puncture process of the second round of seed implantation, the patient developed severe hemoptysis and died of respiratory failure 3 days later. All the other patients underwent no radiation pneumonitis or other serious complications after the second round of iodine-125 seed implantation.

\section{Typical cases}

A 64-year old man (No. 18) with moderately differentiated squamous cell carcinoma $\left(4 \times 5 \times 5 \mathrm{~cm}^{3}\right)$ in the lower right lung and metastasis in the lymph nodes of the mediastinum showed complete response to the combined therapy (Fig. 2). Iodine-125 seeds were implanted to his lung tumor region for brachytherapy; EBRT was used to treat lymph node metastasis. The patient showed complete disappearance of the lung tumor, 2 months
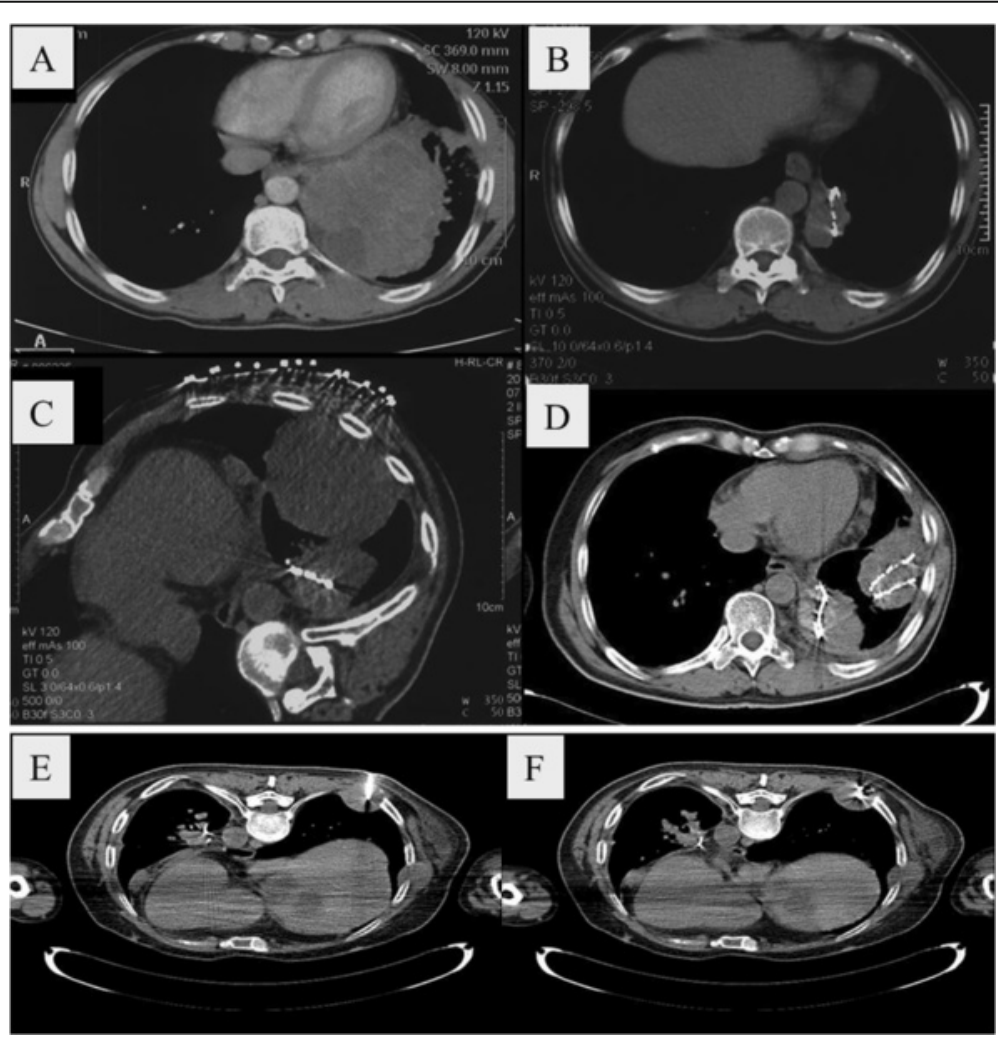

Fig. 3 CT images of a squamous cell lung carcinoma in the lower left lung in a 56-year-old man (No. 3) with brain metastasis: (a) before treatment; (b) 12 months after the first iodine-125 seed implantation of iodine-125 seeds; (c) 30 months after the first implantation showing a recurrent tumor mass $\left(8 \times 4 \mathrm{~cm}^{2}\right)$ in the lower left thoracic cavity; (d) 33 months after the first, i.e. 3 months after the second implantation, showing that the tumor size had decreased by $50 \%$; (e) during the third implantation, i.e., 42 months after the first implantation, showing metastasis on the right side of the chest wall; (f) Immediately after the third implantation 
after implantation. He developed no radiation pneumonia. He was tumor-free for 2 years and is still living.

A 56-year-old man (No. 3) with squamous cell lung carcinoma in the lower left lung and brain metastasis exhibited a significant reduction in tumor size after the first implantation of iodine-125 seeds (Fig. 3). Brain EBRT effectively provided control of the metastasis, while the patient also received 6 cycles of chemotherapy. Tumor size gradually reduced during follow-up and the CT image was tumor-free at the 12-month follow-up. Thirty months after the first round of brachytherapy, a recurrent tumor mass $\left(8 \times 4 \mathrm{~cm}^{2}\right)$ was detected in the lower left thoracic cavity and the patient underwent a second seed implantation procedure. Three months later, the tumor size was reduced by $50 \%$. Forty-two months after the first brachytherapy, metastasis was found on the right side of the chest wall, and the patient received seed implantation for the third time and lived for 4 months afterwards.

A 65-year-old woman (No. 13) had squamous cell lung carcinoma in the upper left lung $\left(7 \times 5 \mathrm{~cm}^{2}\right)$ and metastasis in the superior border of the pericardium and mediastinum $\left(3 \times 4 \mathrm{~cm}^{2}\right)$ (Fig. 4). Due to the large volume of the tumor in the upper left lung, it was hard to keep the volume of normal lung tissue that receiving a radiation dose of 20 Gy (V20) under $30 \%$ when conducting EBRT. Moreover, it was risky to conduct a puncture of the tumor in the mediastinum due to its proximity to large blood vessels. Therefore, seed particles were implanted in the lung tumor while EBRT was delivered to the mediastinum tumor at a dose of $30 \mathrm{~Gy}$. One year after implantation, complete response was achieved. Three years after implantation, no recurrence was observed.

A 57-year-old man (No. 17) had an adenocarcinoma cell lung carcinoma in the lower right lung $\left(3 \times 2 \mathrm{~cm}^{2}\right)$ and metastasis in the vertebra (Fig. 5). Iodine-125 seeds were implanted in both regions and the patient survived for 15 months without further metastasis in the vertebra.

\section{Discussion}

Stage III/IV NSCLC patients with recurrence and metastasis are often inoperable. Palliative radiotherapy and chemotherapy are recommended for these patients, but the efficacy of EBRT and chemotherapy is very limited and the survival time is often short (e.g., 6-9 months). In the present study, EBRT was combined with iodine125 seed implantations for treating locally recurrent
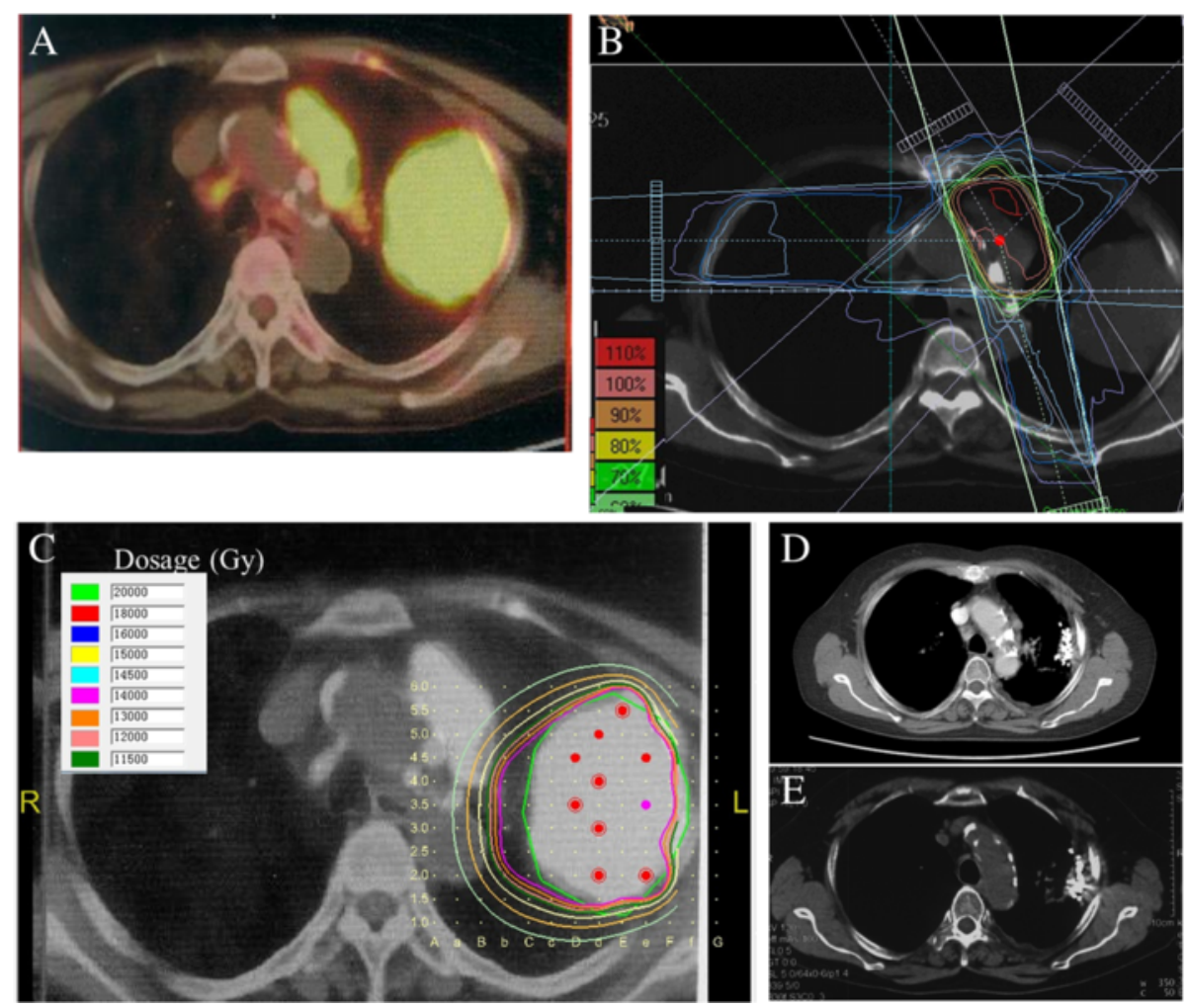

fig. 4 PET-CT image of a massive squamous cell carcinoma $\left(7 \times 5 \mathrm{~cm}^{2}\right)$ in the upper left lung with metastasis in the superior border of the pericardium and mediastinum $\left(3 \times 4 \mathrm{~cm}^{2}\right)$ in a 65 -year-old woman (No. 13) (a). The metastasis region was treated with EBRT at a dose of $30 \mathrm{~Gy}$ (b) and the massive lung cancer region was implanted with iodine-125 seeds (red and purple dots) for brachytherapy (c) guided by CT. Thoracic CT image was tumor-free at 1-year follow-up (d), and showed no recurrence of cancer at 3-year follow-up (e) 

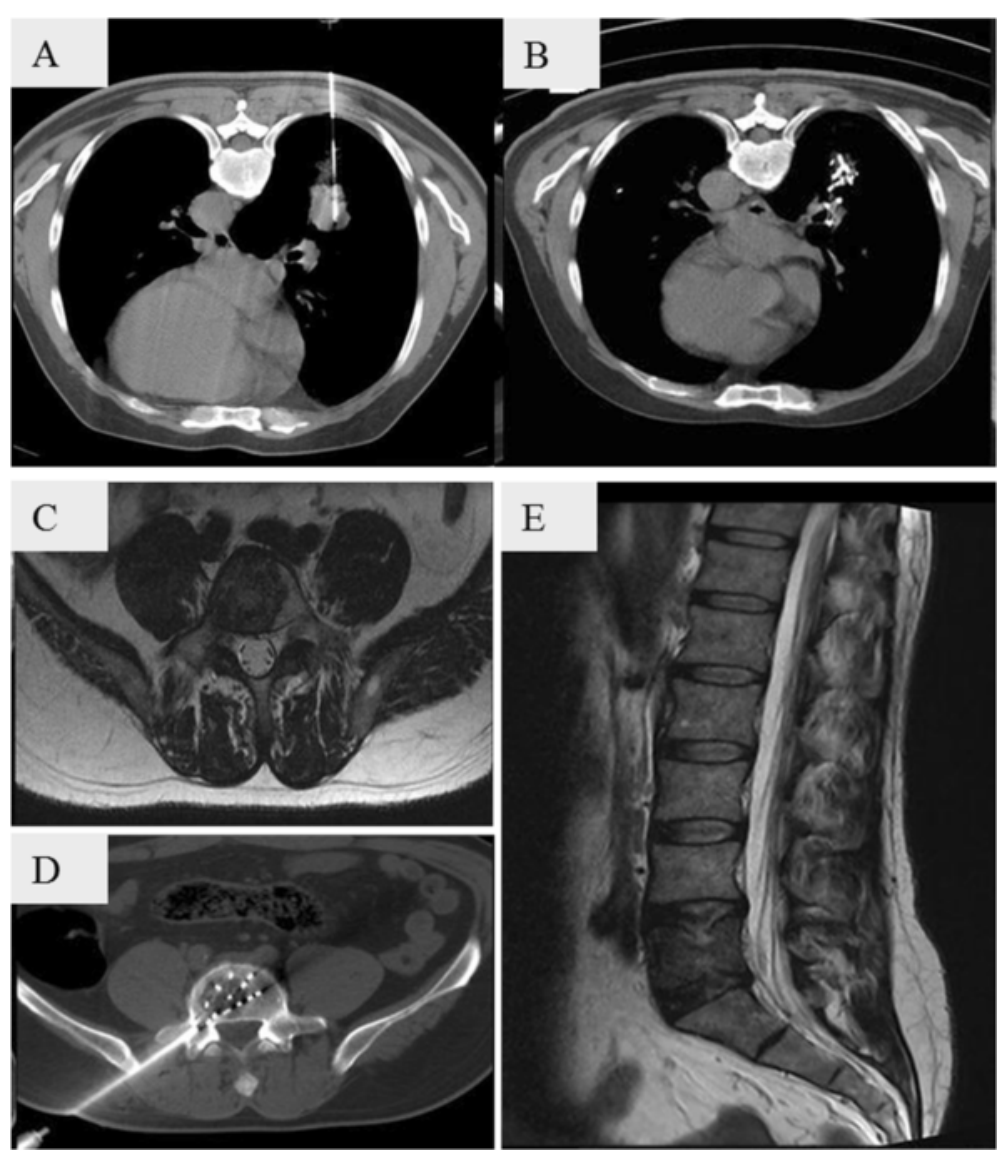

Fig. $5 \mathrm{CT}$ images showing brachytherapy of non-small cell lung adenocarcinoma $\left(3 \times 2 \mathrm{~cm}^{2}\right)$ with vertebral metastasis in a $57-y e a r-o l d ~ m a n ~(N o$. 17). Both the adenocarcinoma region in the lower right lung (a) and the vertebral metastasis region (c, $\mathbf{d}$ ) were implanted with iodine-125 seeds. One-year follow-up images showed good tumor control in both regions $(\mathbf{b}, \mathbf{e})$

NSCLC with concurrent metastasis (patient Nos. 6, 13, 15, 17 and 18). The iodine-125 seed implantations were delivered, at enough radiation doses, to tumors that were difficult to reach by EBRT. We found that repeated iodine-125 seed implantations were beneficial for patients with multiple recurrences, leading to longer median survival time and progression-free survival time, and higher overall 1-year and 2-year survival rates.

For tumors $>5 \mathrm{~cm}$, it is difficult to deliver a large enough dose with EBRT in order to avoid damaging adjacent normal tissues such as the lung, spinal cord, and heart [12]. It has been reported that the effective EBRT dose for tumors $>5 \mathrm{~cm}$ is greater than 100 Gy [3]. However, this high dose of EBRT can lead to an increase in V20 (i.e., more than $30 \%$ of the surrounding normal lung tissue receiving a radiation dose of 20 Gy), thereby increasing the risk of complications. Thus, low doses of EBRT (30-40 Gy) are typically delivered for palliative treatments. In contrast, iodine-125 seed implantation has the potential to deliver a higher radiation dose to a tumor mass (100-140 Gy), and this dose drops off sharply within a short distance. Consequently, adjacent normal tissues are exposed to a minimal dose of radiation, and the short-term risk for serious radiationinduced pneumonitis is reduced [3]. In addition, compared with EBRT, the delivered dose to a tumor by iodine-125 seed implantation can be as much as 2-fold higher [13], with superior treatment efficacy [14]. The response rate reported for EBRT for advanced lung cancer is low, usually below $60 \%$ [14]. In the present study, the response rate for iodine- 125 seed implantation was $88 \%$, consistent with the rates reported in previous studies $[6,15]$.

There are limitations associated with iodine-125 seed implantation. First, it is difficult to perform needle puncture for a metastasis close to the mediastinum, and this may result in an unsatisfactory distribution of iodine125 seeds. Similarly, needle punctures of metastases in the brain or in the lymph nodes of the mediastinum are problematic because of to their proximity to large blood vessels, the heart, or bone structures. The efficacy of iodine-125 seed implantations can also be limited by many factors, such as shielding by bone structures, differences in brachytherapy planning systems, and 
individual differences. Generally, iodine-125 seed implantation is an effective treatment for large tumors. However, its efficacy for poorly differentiated tumors such as small cell lung cancer is poor, due to the low initial dose of iodine- 125 that is applied. Thus, protactinium103, which has a higher initial dose, may be more effective for poorly differentiated tumors such as small cell lung cancer.

Iodine-125 seed implantation in combination with EBRT appears to control recurrent lung cancer and metastasis effectively. For example, the progression-free survival time for the present cohort was 6-8 months, the 1 and 2-year survival rates were 62.5 and $32.7 \%$, and middle survival time of 18 patients was 31 months, respectively. Moreover, these results are an improvement over that have been previously reported $[16,17]$. One reason for this may be because the combined iodine- 125 seed implantation and EBRT provide a better control of the late-stage recurrent lung cancers and metastases in vital organs by increasing the cumulative radiation dose. Another reason may be because that all of in the present study the patients included only experienced restricted metastasis, and patients with diffusive or multiple metastasis were excluded.

\section{Conclusions}

The results of this study demonstrated that iodine- 125 seed implantation in combination with EBRT has the potential to effectively control recurrent and metastatic lung cancer, with less invasiveness, fewer side effects, and higher survival rates. For tumors that are in proximity of the mediastinum or large blood vessels, and thus are inaccessible to puncture, additional EBRT was also beneficial. Thus, iodine- 125 seed implantation in combination with EBRT should be considered an alternative therapy for advanced-stage NSCLC patients. Further studies are needed to confirm this treatment.

\section{Abbreviations}

CT: Computed tomography; EBRT: External beam radiotherapy, EBRT; NSCLC: Non-small cell lung cancer; PET: Positron emission tomography

\section{Acknowledgements}

This manuscript was edited and proofread by Medjaden Bioscience Limited (Hong Kong, P.R China).

\section{Funding}

No funding

\section{Availability of data and materials}

Not applicable.

\section{Authors' contributions}

WL performed data acquisition and drafted the manuscript. DD carried out data processing and statistical analysis. GD and $Y Z$ participated in the design of the study. JJ and XZ assisted in data processing and helped to draft the manuscript. All authors read and approved the final manuscript.

\section{Competing interest}

The authors declare that they have no competing interest.

\section{Consent for publication}

Not applicable.

\section{Ethics approval and consent to participate}

The Institutional Review Board and Ethics Committee of General Hospital of Chengdu Military Region of the PLA (\#08-00125) approved this study. All patients provided written informed consent.

Received: 21 April 2016 Accepted: 19 August 2016

Published online: 13 September 2016

References

1. Siegel R, Naishadham D, Jemal A. Cancer statistics, 2013. CA Cancer J Clin. 2013:63:11-30.

2. Stout R, Barber P, Burt P, Hopwood P, Swindell R, Hodgetts J, et al. Clinical and quality of life outcomes in the first United Kingdom randomized trial of endobronchial brachytherapy (intraluminal radiotherapy) vs. external beam radiotherapy in the palliative treatment of inoperable non- small cell lung cancer. Radiother Oncol. 2000;56:323-7.

3. Reveiz L, Rueda JR, Cardona AF. Palliative endobronchial brachytherapy for non-small cell lung cancer. Cochrane Database System Rev. 2012;12:Cd004284.

4. Wang JJ, Yuan HS, Li JN, Jiang YL, Tian SQ, Yang RJ. CT-guided radioactive seed implantation for recurrent rectal carcinoma after multiple therapy. Med Oncol (Northwood, London, England). 2010;27:421-9.

5. Ceresoli GL, Reni M, Chiesa G, Carretta A, Schipani S, Passoni P, et al. Brain metastases in locally advanced nonsmall cell lung carcinoma after multimodality treatment: risk factors analysis. Cancer. 2002;95:605-12.

6. Wang ZM, Lu J, Liu T, Chen KM, Huang G, Liu FJ. CT-guided interstitial brachytherapy of inoperable non-small cell lung cancer. Lung Cancer (Amsterdam, Netherlands). 2011;74:253-7.

7. Skowronek J. Lung cancer brachytherapy. In: Lung Cancer Treatment. 2011. p. 43-86.

8. Johnson M, Colonias A, Parda D, Trombetta M, Gayou O, Reitz B, Miften M. Dosimetric and technical aspects of intraoperative $1-125$ brachytherapy for stage I non-small cell lung cancer. Phys Med Biol. 2007;52(5):1237-45.

9. Huang Q, Chen J, Chen Q, Lai Q, Cai S, Luo K, et al. Computed tomographic-guided iodine-125 interstitial implants for malignant thoracic tumors. Euro J Radiol. 2013;82:2061-6.

10. Jiang G, Li Z, Ding A, Zhou F, Jiao W, Tang D, et al. Computed tomographyguided iodine-125 interstitial implantation as an alternative treatment option for lung cancer. Indian J Cancer. 2015;51 Suppl 2:e9-12.

11. Monk BJ, Tewari KS, Puthawala AA, Syed AM, Haugen JA, Burger RA. Treatment of recurrent gynecologic malignancies with iodine-125 permanent interstitial irradiation. Int J Radiat Oncol Biol Phys. 2002;52:806-15.

12. Li W, Guan J, Yang L, Zheng X, Yu Y, Jiang J. lodine-125 brachytherapy improved overall survival of patients with inoperable stage III/IV non-small cell lung cancer versus the conventional radiotherapy. Med Oncol. 2014; 32(1):395

13. Timmerman R, McGarry R, Yiannoutsos C, Papiez L, Tudor K, DeLuca J, et al. Excessive toxicity when treating central tumors in a phase II study of stereotactic body radiation therapy for medically inoperable early-stage lung cancer. J Clin Oncol. 2006;24:4833-39.

14. Ragde H, Elgamal AAA, Snow PB, Brandt J, Bartolucci AA, Nadir BS, et al. Ten-year disease free survival after transperineal sonography-guided iodine125 brachytherapy with or without 45-gray external beam irradiation in the treatment of patients with clinically localized, low to high gleason grade prostate carcinoma. Cancer. 1998;83:989-1001.

15. Zhang S, Zheng Y, Yu P, Yu F, Zhang Q, Lv Y, et al. The combined treatment of CT-guided percutaneous 1251 seed implantation and chemotherapy for non-small-cell lung cancer. J Cancer Res Clin Oncol. 2011;137:1813-22.

16. Lee W, Daly BD, DiPetrillo TA, Morelli DM, Neuschatz AC, Morr J, et al. Limited resection for non-small cell lung cancer: observed local control with implantation of I-125 brachytherapy seeds. Ann Thorac Surg. 2003;75:237-42. discussion 233-42.

17. Ung YC, Yu E, Falkson C, Haynes AE, Stys-Norman D, Evans WK. The role of high-dose-rate brachytherapy in the palliation of symptoms in patients with non-small-cell lung cancer: a systematic review. Brachytherapy. 2006;5:189-202. 